PROCEEDINGS OF THE AMERICAN MATHEMATICAL SOCIETY

Volume 124, Number 7, July 1996

\title{
REAL INSTANTONS, DIRAC OPERATORS AND QUATERNIONIC CLASSIFYING SPACES
}

\author{
PAUL NORBURY AND MARC SANDERS \\ (Communicated by Ronald Stern)
}

\begin{abstract}
Let $M(k, S O(n))$ be the moduli space of based gauge equivalence classes of $S O(n)$ instantons on principal $S O(n)$ bundles over $S^{4}$ with first Pontryagin class $p_{1}=2 k$. In this paper, we use a monad description (Y. Tian, The Atiyah-Jones conjecture for classical groups, preprint, S. K. Donaldson, Comm. Math. Phys. 93 (1984), 453-460) of these moduli spaces to show that in the limit over $n$, the moduli space is homotopy equivalent to the classifying space $B S p(k)$. Finally, we use Dirac operators coupled to such connections to exhibit a particular and quite natural homotopy equivalence.
\end{abstract}

\section{INTRODUCTION}

Let $M(k, S O(n))$ be the moduli space of based gauge equivalence classes of $S O(n)$ instantons on principal $S O(n)$ bundles over $S^{4}$ with first Pontryagin class $p_{1}=2 k$. By adding a trivial connection on a trivial line bundle, there are natural maps $M(k, S O(n)) \hookrightarrow M(k, S O(n+1))$, and one can define the direct limit space $M(k, S O)$. In this paper we show that there is a homotopy equivalence $M(k, S O) \simeq B S p(k)$, where $S p(k)$ denotes the symplectic group of norm preserving automorphisms of the quaternionic vector space $H^{k}$. We also show that this equivalence can be realized by a "Dirac-type" map, constructed by coupling a Dirac operator to an $S O(n)$ connection. More precisely, the coupling of a Dirac operator to a connection associates to each element of $M(k, S O(n))$ an operator acting on the space of sections of a certain vector bundle. Associated to each selfdual connection is the vector space of sections in the kernel of its associated operator. This procedure defines a complex vector bundle, which for $S O(n)$ connections has a symplectic structure, and this bundle is classified by a map which we shall refer to as the Dirac map, $\partial_{S O(n)}: M(k, S O(n)) \rightarrow B S p(k)$. The topological properties of these Dirac maps for $S U(n)$ connections were first studied by Atiyah and Jones [AJ], and more recently it was shown in [S] that the limit map $\partial_{S U}: M(k, S U) \rightarrow B U(k)$ realizes Kirwan's $[\mathrm{K}]$ homology isomorphism $H_{*}(M(k, S U)) \cong H_{*}(B U(k))$, and is, therefore, a homotopy equivalence. It also makes sense to define such Dirac maps on the limit spaces $M(k, G)$, where $G$ is either $S O$ or $S p$, and in [S] it was shown that the limit map $\partial_{S p}: M(k, S p) \rightarrow B O(k)$ is a homotopy equivalence. In this

Received by the editors January 13, 1995.

1991 Mathematics Subject Classification. Primary 53C07, 55P38; Secondary 55R45.

Key words and phrases. Instantons, Dirac operators, classifying space.

(C)1996 American Mathematical Society 
paper we complete the picture for the classical groups by showing that the limit map $\partial_{S O}: M(k, S O) \rightarrow B S p(k)$ is also a homotopy equivalence.

Our proof will be fairly direct. In Section 1 we review Tian's [Ti] version of Donaldson's [D] monad description of $M(k, S O(n))$. Tian exhibits this moduli space as the quotient of a set of triples of certain complex matrices by an action of $S p(k ; C)$, the complex symplectic group. We shall show that this action is free, that there are natural maps $M(k, S O(n)) \hookrightarrow M(k, S O(n+1))$, and that in the limit over $n$ the space of triples is contractible. Hence, $M(k, S O)$ will be shown to be the quotient of a contractible space by a free $S p(k ; C)$ action. In Section 2 , we use a comparison between $S O(n)$ and $S U(n)$ connections to show that the Dirac map $\partial_{S O(n)}: M(k, S O(n)) \rightarrow B S p(k)$ induces a surjection in integral homology through a range of dimension increasing with $n$. Since $H_{*}(M(k, S O) ; Z) \cong H_{*}(B S p(k) ; Z)$ by results of Section 1, the limit map $\partial_{S O}$ must be a homology isomorphism and therefore a homotopy equivalence.

Notice that the $S p$ and $S O$ duality in these moduli spaces is foreshadowed in Bott Periodicity. Since the entire space of based gauge equivalence classes of $S O(n)$ connections is homotopy equivalent to $\Omega^{3} S O(n)$, the limit over $n$ is homotopy equivalent to $Z \times B S p$. Similarly, the space of $S p(n)$ connections is homotopy equivalent to $\Omega^{3} S p(n)$ which, after passing to the limit, is homotopy equivalent to $Z \times B O$. Alternatively, as we will see in Section 2, this duality comes from the fact that the bundle of real spinors over $S^{4}$ is naturally a symplectic vector bundle. Recently, in fact, Tian [Ti] has shown that by comparing the two possible limit processes which one can apply to these moduli spaces, viz., fixing $k$ and taking the limit over $n$ or fixing $n$ and taking the limit over $k$, one actually can prove Bott Periodicity. This consequence alone demonstrates the beauty and complexity of these moduli spaces.

\section{2. $M(k, S O)$ AND $B S p(k)$}

The ADHM construction [ADHM] identifies the space of instantons with certain holomorphic bundles over complex projective space, and Donaldson [D] used a monad construction to characterize such bundles in terms of a quotient of a set of sequences of complex matrices by a natural group action. For $S O(n)$ instantons, Tian [Ti] carried out this procedure explicitly.

Let $\sigma$ denote the standard skew form on $\mathbf{C}^{2 k}$,

$$
\sigma=\left(\begin{array}{cc}
0 & I_{k} \\
-I_{k} & 0
\end{array}\right)
$$

where $I_{k}$ is the $k \times k$ identity matrix. The complexified symplectic group, $S p(k, \mathbf{C}) \subset$ $G l(2 k, \mathbf{C})$, consists of those matrices $g$ such that $g^{-1}=-\sigma g^{T} \sigma$. The maximal compact subgroup of $S p(k, \mathbf{C})$ is the compact symplectic group $S p(k)$.

Proposition 1 (Donaldson [D] and Tian [Ti]). Let $A(k, S O(n))$ be the space of triples of complex matices $\left(\gamma_{1}, \gamma_{2}, c\right)$, where $\gamma_{i}$ is $2 k \times 2 k$ and $c$ is $n \times 2 k$, satisfying:

a) $\gamma_{1}^{T}=-\sigma \gamma_{1} \sigma$,

b) $\gamma_{2}^{T}=-\gamma_{2}$,

c) $2\left(\gamma_{1}^{T} \gamma_{2}+\gamma_{2}^{T} \gamma_{1}\right)+c^{T} c=0$,

d) $\left(\begin{array}{c}\gamma_{1}+x I_{2 k} \\ \gamma_{2}+y \sigma \\ c\end{array}\right)$ has rank $2 k$ for all $x, y \in \mathbf{C}$. 
Then there is a natural action of $S p(k, \mathbf{C})$ on $A(k, S O(n))$ given by

$$
g \cdot\left(\gamma_{1}, \gamma_{2}, c\right)=\left(g \gamma_{1} g^{-1},\left(g^{-1}\right)^{T} \gamma_{2} g^{-1}, c g^{-1}\right),
$$

and $M(k, S O(n))$ is homeomorphic to the quotient $A(k, S O(n)) / S p(k, \mathbf{C})$.

It has been shown $[\mathrm{S}]$ that in the analogous description of $S U(n)$ and $S p(n)$ instantons, this group action is free. This is, in some sense, already implicit in the monad construction. Not surprisingly, then, it is also true in the $S O(n)$ case. For the sake of completeness, however, we now give the proof.

Lemma 2. The natural action of $S p(k, \mathbf{C})$ on $A(k, S O(n))$ is free.

Proof. Assume the converse, so we have

$$
\left(g \gamma_{1} g^{-1},\left(g^{-1}\right)^{T} \gamma_{2} g^{-1}, c g^{-1}\right)=\left(\gamma_{1}, \gamma_{2}, c\right)
$$

for a particular $g \neq I$ and triple $\left(\gamma_{1}, \gamma_{2}, c\right)$, and note that elements of $S p(k, \mathbf{C})$ satisfy $g^{-1}=-\sigma g^{T} \sigma$. Consider the subspace $i m(g-I)=V \subset \mathbf{C}^{2 k}$. By assumption it is non-empty. Thus, from the definition of the action we have

$$
\begin{gathered}
c(g-I)=0, \\
\gamma_{1}(g-I)=(g-I) \gamma_{1}, \\
\sigma \gamma_{2}(g-I)=(g-I) \sigma \gamma_{2} .
\end{gathered}
$$

This last fact is proved as follows:

$$
\begin{aligned}
\gamma_{2} & =\left(g^{-1}\right)^{T} \gamma_{2} g^{-1} \\
\Rightarrow \gamma_{2} g & =\left(g^{-1}\right)^{T} \gamma_{2} \\
\Rightarrow \sigma \gamma_{2} g & =-\sigma\left(g^{-1}\right)^{T} \sigma \sigma \gamma_{2} \\
\Rightarrow \sigma \gamma_{2} g & =g \sigma \gamma_{2} .
\end{aligned}
$$

Equivalently $c$ annihilates $V$ and $\gamma_{1}$ and $\sigma \gamma_{2}$ preserve $V$. Using conditions a), b), and c), we see that on $V$

$$
\begin{aligned}
& \gamma_{1}^{T} \gamma_{2}+\gamma_{2}^{T} \gamma_{1}=0 \\
\Rightarrow & -\sigma \gamma_{1} \sigma \gamma_{2}-\gamma_{2} \gamma_{1}=0 \\
\Rightarrow & \gamma_{1} \sigma \gamma_{2}-\sigma \gamma_{2} \gamma_{1}=0 .
\end{aligned}
$$

Hence $\gamma_{1}$ and $\sigma \gamma_{2}$ have a common eigenvector in $V$.

Choose $v \in V$ satisfying $\gamma_{1} v=\lambda v$ and $\sigma \gamma_{2} v=\mu v$. Then

$$
\left(\begin{array}{c}
\gamma_{1}-\lambda I_{2 k} \\
\gamma_{2}+\mu \sigma \\
c
\end{array}\right) v=0
$$

contradicting condition d). Thus, the image of $g-I$ must be empty, so $g=I$ and the action is free.

We now construct the limit space $M(k, S O)$ and show that it is homotopy equivalent to $B S p(k)$. First notice that there is an $S p(k, \mathbf{C})$ equivariant map from $A(k, S O(n)) \hookrightarrow A(k, S O(n+1))$ which sends each $\gamma_{i}$ to itself and sends $c$ to the $(n+1) \times k$ matrix made up of $c$ with an extra row of zeros on top. On the level of monads, this adds to the bundle over $C P^{2}$ the trivial holomorphic line bundle (see [Ti]). Thus this map induces the natural inclusion $M(k, S O(n)) \hookrightarrow M(k, S O(n+1))$ sending the connection $\omega$ to the connection $\omega \oplus d$, where $d$ is ordinary exterior differentiation. We now prove the main theorem of this section. 
Theorem 3. $A(k, S O)$ is a contractible space with a free $S p(k, \mathbf{C})$ action. Thus, $M(k, S O) \cong A(k, S O) / S p(k, \mathbf{C}) \simeq B S p(k)$.

Proof. To show that $A(k, S O)$ is contractible it suffices to show that all of its homotopy groups are zero. To this end we show that for any $k$ and $n$ there is an $r>n$ such that inclusion $A(k, S O(n)) \hookrightarrow A(k, S O(r))$ is homotopically trivial (cf. [S], sections 2 and 3$)$.

For $0 \leq t \leq 1$ define $\tilde{I}_{k}(t)$ to be the $4 k \times 2 k$ matrix whose $j^{\text {th }}$ column is the vector

$$
\left(\begin{array}{c}
0 \\
\vdots \\
0 \\
t \\
i t \\
0 \\
\vdots \\
0
\end{array}\right)
$$

where there are $2 j-2$ zeroes before the $t$. Note that $\left(\tilde{I}_{k}(t)\right)^{T} \cdot \tilde{I}_{k}(t)$ is the zero matrix. Now consider the homotopy $H_{t}: A(k, S O(n)) \rightarrow A(k, S O(4 k+n))$ defined as follows:

$$
H_{t}\left(\gamma_{1}, \gamma_{2}, c\right)=\left((1-t) \gamma_{1},(1-t) \gamma_{2}, c_{t}\right)
$$

where

$$
c_{t}=\left(\begin{array}{c}
\tilde{I}_{k}(t) \\
(1-t) c
\end{array}\right) .
$$

It is easy to check that for any $x \in A(k, S O(n))$ we have $H_{t}(x) \in A(k, S O(4 k+n))$ because $c_{t}$ clearly has rank $2 k$ and $c_{t}^{T} \cdot c_{t}=c^{T} \cdot c(1-t)^{2}$. Finally, notice that $H_{0}$ is just the natural inclusion $A(k, S O(n)) \hookrightarrow A(k, S O(4 k+n))$, and $H_{1}$ is a constant map. This finishes the proof of the theorem.

\section{The Dirac MAP $\partial_{S O(n)}: M(k, S O(n)) \rightarrow B S p(k)$}

In this section we review the construction of the Dirac map, and show that after passing to the limit over $n$ it is a homotopy equivalence. To define this map it is instructive to first consider $S U(n)$ instantons. Let $\omega$ be a connection on the $S U(n)$ vector bundle $E_{k}$, where the second Chern class $c_{2}\left(E_{k}\right)=k$, and let $S$ denote the canonical bundle of complex spinors over $S^{4}$ with its canonical connection $\nabla_{s}$. The tensor product bundle $S \otimes E_{k}$ inherits a Clifford module structure from the one on $S$, and we can view $\nabla_{s} \otimes \omega$ as a connection on this bundle. This connection gives rise to a Dirac operator

$$
D_{\omega}: \Gamma\left(S \otimes E_{k}\right) \longrightarrow \Gamma\left(S \otimes E_{k}\right),
$$

where $\Gamma\left(S \otimes E_{k}\right)$ is the space of smooth sections of $S \otimes E_{k}$. There is a splitting $S \cong S^{+} \oplus S^{-}$and the Dirac operator interchanges the two summands. The operator

$$
D_{\omega}^{+}: \Gamma\left(S^{+} \otimes E_{k}\right) \longrightarrow \Gamma\left(S^{-} \otimes E_{k}\right)
$$

is Fredholm, in an appropriate Sobolev completion, and of index $k$ [AJ]. Furthermore, if $\omega$ is selfdual, then $\operatorname{Coker}\left(D_{\omega}^{+}\right)=0$ [AHS]. Therefore, the space of sections in the kernel of $D_{\omega}^{+}$gives a well-defined vector space associated to the connection 
$\omega$. There is an equivariance of the kernel under gauge transformation in the sense that $\sigma \in \operatorname{Ker}\left(D_{\omega}^{+}\right)$implies $g \sigma \in \operatorname{Ker}\left(D_{g w}^{+}\right)$, for any $g$ in the based gauge group of bundle automorphisms of $E_{k}$. Passing to gauge equivalence classes gives a $k$ dimensional complex vector bundle over $M(k, S U(n))$. This bundle is classified by a map, $\partial_{S U(n)}: M(k, S U(n)) \rightarrow B U(k)$, which we shall refer to as the Dirac map.

A similar construction can be used to define the Dirac map for $S O(n)$ connections. Given an $S O(n)$ bundle $E$ with $p_{1}(E)=2 k$ and an $S O(n)$ instanton $\omega$ on $E$, we can complexify the bundle and connection, denoted $\omega_{C}$ and $E_{C}$, and then use the unitary Dirac map to obtain

$$
M(k, S O(n)) \longrightarrow M(2 k, S U(n)) \stackrel{\partial_{S U(n)}}{\longrightarrow} B U(2 k)
$$

(Note that $c_{2}\left(E_{C}\right)=2 k$.) However, because $E_{C}$ has by definition an underlying real structure, given by some bundle involution $J_{E}$, and the complex spinor bundle $S$ has a quaternionic structure, given by some complex anti-linear bundle automorphism $J_{s}$, where $J_{s} \circ J_{s}=-1$, the tensor product bundle $S \otimes E_{C}$ will also have a quaternionic structure. Moreover, the Dirac operator will respect this extra structure because the tensor product connection $\nabla_{s} \otimes \omega_{C}$ will commute with $J_{s} \otimes J_{E}$. Thus, the kernel bundle, defined by coupling a Dirac operator to a real $S O(n)$ instanton, will be a $k$-dimensional quaternionic bundle over $M(k, S O(n))$. In other words, the composition

$$
M(k, S O(n)) \longrightarrow M(2 k, S U(n)) \stackrel{\partial_{S U(n)}}{\longrightarrow} B U(2 k)
$$

factors through $B S p(k)$. We denote this lifting by $\partial_{S O(n)}$. In short, we have the homotopy commutative diagram

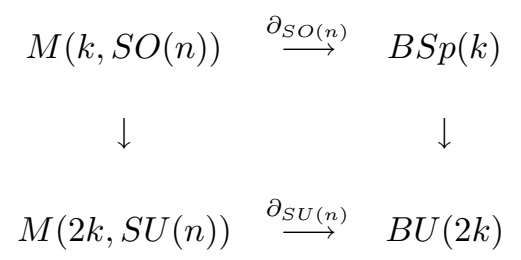

We now show that we can define the limit map $\partial_{S O}: M(k, S O) \rightarrow B S p(k)$. From the matrix description of $M(k, S O(n))$, we see that the natural inclusion $M(k, S O(n)) \hookrightarrow M(k, S O(n+1))$, mapping $(\omega, E)$ to $(\omega \oplus d, E \oplus R)$, embeds $M(k, S O(n))$ as a closed submanifold of $M(k, S O(n+1))$. It follows that the direct limit $M(k, S O)$ is homotopy equivalent to the homotopy direct limit $M(k, S O)_{h}$. Thus, it suffices to define $\partial_{S O}$ on $M(k, S O)_{h}$. To this end, let $\mathcal{A}(k, S O(n))$ denote the space of instantons before passing to gauge equivalence classes, and let $G_{k, S O(n)}$ denote the based gauge group of bundle automorphisms of the $S O(n)$ bundle $E$, where $p_{1}(E)=2 k$. Let $\eta(k, S O(n))$ denote the bundle classified by the map $\partial_{S O(n)}$ : $M(k, S O(n)) \rightarrow B S p(k)$. By definition,

$$
\eta(k, S O(n))=\left\{[(\omega, \tau)]: \tau \in \operatorname{ker}\left(D_{\omega}^{+}\right)\right\} \subset \mathcal{A}(k, S O(n)) \times_{G_{k, S O(n)}} \Gamma\left(S^{+} \otimes E_{C}\right) .
$$


Since the untwisted Dirac operator on $S^{4}$ has no kernel $\left(S^{4}\right.$ has no harmonic spinors), the natural inclusion of bundles

$$
\begin{array}{cccc}
\eta(k, S O(n)) & \hookrightarrow & \eta(k, S O(n+1)) \\
\downarrow & & \downarrow \\
M(k, S O(n)) & \hookrightarrow & M(k, S O(n+1))
\end{array}
$$

defined by $(\omega, \tau) \rightarrow(\omega \oplus d, \tau \oplus 0)$ is an isomorphism on fibers. Thus the pullback of $\eta(k, S O(n+1))$ via the inclusion $M(k, S O(n)) \hookrightarrow M(k, S O(n+1))$ is isomorphic to $\eta(k, S O(n))$. Hence, the diagram

$$
\begin{array}{ccc}
M(k, S O(n)) & \hookrightarrow & M(k, S O(n+1)) \\
\partial_{S O(n) \downarrow} \downarrow & & \downarrow \partial_{S O(n+1)} \\
B S p(k) & = & B S p(k)
\end{array}
$$

commutes up to homotopy. So there exists a map $\partial_{S O}: M(k, S O)_{h} \rightarrow B S p(k)$. Precomposing with the equivalence $M(k, S O) \simeq M(k, S O)_{h}$ gives a map

$$
\partial_{S O}: M(k, S O) \rightarrow B S p(k) .
$$

This map is not necessarily uniquely determined. Nevertheless, any two choices, when restricted to $M(k, S O(n))$, will classify the bundle $\eta(k, S O(n))$, and this is the only property of the limit map which we will use. In particular, any such choice will give a homotopy commutative diagram

$$
\begin{aligned}
& M(k, S O(n)) \longrightarrow M(k, S O) \\
& \partial_{S O(n)} \searrow \quad \downarrow \partial_{S O} \\
& B S p(k) .
\end{aligned}
$$

Now, since $H_{*}(M(k, S O)) \cong H_{*}(B S p(k))$ by Theorem $3, \partial_{S O}$ will induce a homology isomorphism, and therefore be a homotopy equivalence, if and only if it induces a surjection in homology. By the homotopy commutativity of the previous diagram, it suffices to show that $\partial_{S O(n)}: M(k, S O(n)) \rightarrow B S p(k)$ induces a surjection in homology through a range increasing with $n$.

Theorem 4. The Dirac map $\partial_{S O(n)}$ induces a surjection in homology through dimension $2 n-4$. Thus, the limit map $\partial_{S O}: M(k, S O) \rightarrow B S p(k)$ is a homotopy equivalence.

We begin by proving the following lemma:

Lemma 5. There is a commutative diagram

$$
\begin{array}{ccc}
H_{*}(M(k, S U(n))) & \stackrel{\left(\partial_{S U(n)}\right)_{*}}{\longrightarrow} & H_{*}(B U(k)) \\
\downarrow & \downarrow \\
H_{*}(M(k, S O(2 n))) & \stackrel{\left(\partial_{S O(2 n)}\right)_{*}}{\longrightarrow} & H_{*}(B S p(k)),
\end{array}
$$


where $S p(k) \subset U(2 k)$ consists of all matrices of the form

$$
\left(\begin{array}{cc}
A & B \\
-\bar{B} & \bar{A}
\end{array}\right)
$$

for any $A, B \in \operatorname{End}\left(C^{k}\right)$, and the map $B U(k) \rightarrow B S p(k)$ is induced from the inclusion $U(k) \hookrightarrow S p(k)$ defined by

$$
A \mapsto\left(\begin{array}{cc}
A & 0 \\
0 & \bar{A}
\end{array}\right)
$$

Proof. First notice that the natural map of Lie algebras $s u(n) \hookrightarrow s o(2 n)$ induces a map $M(k, S U(n)) \rightarrow M(k, S O(2 n))$. The self-duality condition is preserved because the Hodge star operator is complex linear. Locally, the connection matrix $\gamma=\gamma_{1}+i \gamma_{2}$, where $\gamma_{j}$ is a real matrix-valued one form, will map to the matrix

$$
\left(\begin{array}{cc}
\gamma_{1} & -\gamma_{2} \\
\gamma_{2} & \gamma_{1}
\end{array}\right)
$$

Also notice that, as mentioned previously, the complexification of a real connection on an $S O(r)$ bundle induces a natural map $M(k, S O(2 n)) \rightarrow M(2 k, S U(2 n))$. Locally, the composition of these two maps

$$
M(k, S U(n)) \rightarrow M(k, S O(2 n)) \rightarrow M(2 k, S U(2 n))
$$

is given by

$$
\gamma=\gamma_{1}+i \gamma_{2} \mapsto\left(\begin{array}{cc}
\gamma_{1} & -\gamma_{2} \\
\gamma_{2} & \gamma_{1}
\end{array}\right) \mapsto\left(\begin{array}{cc}
\gamma_{1} & -\gamma_{2} \\
\gamma_{2} & \gamma_{1}
\end{array}\right),
$$

where the last matrix is viewed as taking values in the Lie algebra $s u(2 n)$. Since there is a $g \in S U(2 n)$ such that

$$
g^{-1}\left(\begin{array}{cc}
\gamma_{1} & -\gamma_{2} \\
\gamma_{2} & \gamma_{1}
\end{array}\right) g=\left(\begin{array}{cc}
\gamma_{1}+i \gamma_{2} & 0 \\
0 & \gamma_{1}-i \gamma_{2}
\end{array}\right)=\left(\begin{array}{cc}
\gamma & 0 \\
0 & \bar{\gamma}
\end{array}\right),
$$

the connections represented by these matrix-valued one forms are gauge equivalent. Thus, the composition $M(k, S U(n)) \rightarrow M(k, S O(2 n)) \rightarrow M(2 k, S U(2 n))$ sends the equivalence class of the selfdual connection $\omega$ on the bundle $E$ to the equivalence class of the selfdual connection $\omega \oplus \bar{\omega}$ on the bundle $E \oplus \bar{E}$.

Now consider the diagram

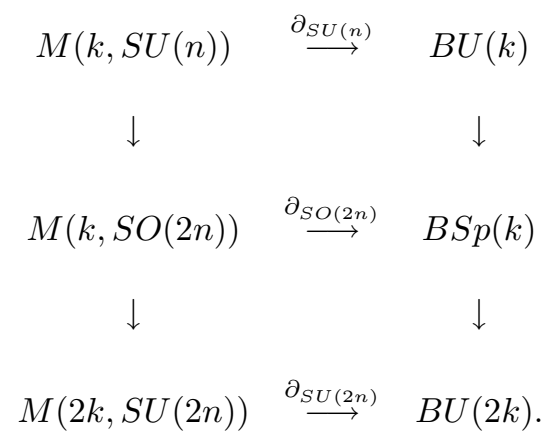


By the definition of $\partial_{S O(2 n)}$, the bottom square homotopy commutes. Since the map $B S p(k) \rightarrow B U(2 k)$ induces an injection in homology, the top square will induce a commutative diagram in homology if the large outer "square"

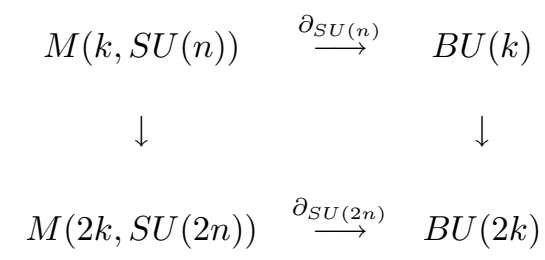

commutes in homology. Note that on the level of bundles the right vertical map sends a complex vector bundle $F$ to the complex bundle $F \oplus \bar{F}$. Let $\eta(r, S U(l))$ denote the Dirac bundle classified by the map $\partial_{S U(l)}: M(r, S U(l)) \rightarrow B U(r)$. The proof of the lemma will be complete if the composition

$$
M(k, S U(n)) \longrightarrow M(2 k, S U(2 n)) \stackrel{\partial_{S U(2 n)}}{\longrightarrow} B U(2 k)
$$

classifies the bundle $\eta(k, S U(n)) \oplus \bar{\eta}(k, S U(n))$. There is a natural bundle map

$$
\begin{array}{ccc}
\eta(k, S U(n)) \oplus \bar{\eta}(k, S U(n)) & \longrightarrow & \eta(2 k, S U(2 n)) \\
\downarrow & & \downarrow \\
M(k, S U(n)) & \longrightarrow & M(2 k, S U(2 n))
\end{array}
$$

defined by

$$
\left[\left(\omega, \psi_{1} \oplus \psi_{2}\right)\right] \mapsto\left[\left(\omega \oplus \bar{\omega}, \psi_{1} \oplus \bar{\psi}_{2}\right)\right]
$$

where $\bar{\psi}_{2}$ is the section $\psi_{2}$ viewed as a section of the conjugate bundle. Since $\psi$ is in the kernel of $D_{\omega}^{+}$if and only if $\bar{\psi}$ is in the kernel of $D_{\bar{\omega}}^{+}$, this bundle map is a surjection on fibers. Since the fibers have the same dimension, this map is an isomorphism. Thus $\eta(k, S U(n)) \oplus \bar{\eta}(k, S U(n))$ is isomorphic to the pullback of $\eta(2 k, S U(2 n))$, and the lemma is proved.

The proof of Theorem 4 is now easy. In $[\mathrm{S}]$, section 5 , it was shown that the map

$$
\left(\partial_{S U(n)}\right)_{*}: H_{*}(M(k, S U(n))) \longrightarrow H_{*}(B U(k))
$$

is a surjection through dimension $2 n-4$. Furthermore, we know that the map $B U(k) \rightarrow B S p(k)$ induces a surjection in homology. Thus, by the commutativity of the diagram

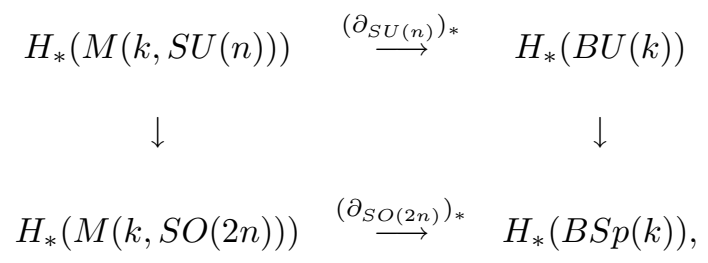

$\left(\partial_{S O(2 n)}\right)_{*}: H_{*}(M(k, S O(2 n))) \rightarrow H_{*}(B S p(k))$ must also be a surjection through this range. In particular, then, the limit map $\partial_{S O}: M(k, S O) \rightarrow B S p(k)$ is a homotopy equivalence. 


\section{REFERENCES}

[ADHM] M.F. Atiyah, V. G. Drinfeld, N.J. Hitchin and Y. I. Manin, Construction of instantons, Phys. Lett. A, 65 (1978), 185-187. MR 82g:81049

[AJ] M.F. Atiyah and J.D.S. Jones, Topological aspects of Yang-Mills theory, Comm. Math. Phys. 61 (1978), 97-118. MR 80j:58021

[AHS] M.F. Atiyah, N.J. Hitchin, and I.M. Singer, Self-duality in four dimensional Riemannian Geometry, Proc. of Royal Society London, A 362 (1978), 425-461. MR 80d:53023

[D] S.K. Donaldson, Instantons and geometric invariant theory, Comm. Math. Phys., 93 (1984), 453-460. MR 86m:32043

[K] F. Kirwan, Geometric Invariant Theory and the Atiyah-Jones conjecture, Proceedings of the Sophus Lie Memorial Conference (Oslo, 1992), editors O.A. Laudal and B. Jahren, Scandinavian University Press, 1994, 161-188.

[S] M. Sanders, Classifying spaces and Dirac operators coupled to instantons, Transactions of the American Mathematical Society 347 (1995), 4037-4072. CMP 95:15

[Ti] Y. Tian, The Atiyah-Jones Conjecture for Classical Groups, preprint.

Mathematics Institute, University of Warwick, Coventry CV4 7AL, United Kingdom E-mail address: norbs@maths.warwick.ac.uk

Department of Mathematics, University of Minnesota, Minneapolis, Minneapolis, MinNESOTA 55455

E-mail address: sanders@math.umn.edu 